\title{
COPD patients' ability to follow exercise influences short-term outcomes of rehabilitation
}

\author{
M.A. Puhan*, H.J. Schünemann ${ }^{\#, \uparrow_{,+}}$, G. Buesching ${ }^{\S}$, E. vanOort ${ }^{\S}$, \\ A. Spaar* and M. Frey ${ }^{\S}$
}

ABSTRACT: Chronic obstructive pulmonary disease (COPD) patients with limited ability to follow exercise protocols may receive smaller benefits from rehabilitation. The association between the ability to follow exercise protocols and short-term outcomes of rehabilitation was assessed in COPD patients.

As a measure of the ability to follow exercise protocols, the number of major breaks lasting $\geqslant 1$ min was determined in 98 COPD patients during supervised exercise sessions. The benefits from rehabilitation were compared between patients with, on average, more than one and one or fewer major breaks per session.

Patients with one or fewer major breaks per session showed significantly greater improvements in exercise capacity (between-groups difference of $38 \mathrm{~m}$ for 6-min walking distance, $22.1 \mathrm{~W}$ for short-time maximum exercise capacity (steep ramp test) and $5.5 \mathrm{~W}$ for maximum exercise capacity). Quality of life also tended to be better in patients with one or fewer major breaks per session, but the differences were nonsignificant (adjusted between-groups difference in Chronic Respiratory Disease Questionnaire total score of 0.14 ).

Limited ability to follow exercise protocols is associated with smaller benefits of rehabilitation. This finding highlights the importance of choosing tolerable exercise protocols for chronic obstructive pulmonary disease patients.

KEYWORDS: Chronic obstructive pulmonary disease, exercise, exercise test, quality of life, respiratory rehabilitation

P hysical exercise plays a critical role in chronic obstructive pulmonary disease (COPD) management [1-3]. It improves health-related quality of life (HRQoL) and is likely to reduce the risk of hospital admissions and death [4, 5]. However, patients with advanced COPD are often limited by dyspnoea and fatigue, rendering physical exercise difficult. Since the 1990s, great research efforts have been made to develop tolerable but effective exercise protocols [6].

Continuous and interval exercise have become the most widely applied protocols for lower limb training in COPD patients [4, 6]. During continuous exercise, patients exercise at a constantly high workload of $\sim 70 \%$ of maximum exercise capacity. During interval exercise, patients exercise alternately at high and low workload in order to allow them to recover intermittently from the high-workload intervals. It is thought that a lower degree of dynamic hyperinflation results from interval exercise than continuous exercise, which is associated with good tolerance of the interval exercise modality [7-9]. Various investigators have shown, in randomised trials, that interval exercise is not less effective than continuous exercise at improving the HRQoL and exercise capacity of patients with COPD [10-14].

Little attention has been paid to the ability of patients to follow specific exercise protocols and whether this has consequences for the short-term outcomes of respiratory rehabilitation. If an exercise protocol is tolerated well during the supervised initiation stage of exercise, it may motivate patients to continue their training and increase attendance of exercise sessions. These questions have not been addressed empirically. Therefore, the aim of the present study was to assess the ability of patients with severe COPD to follow continuous and interval exercise protocols, and to explore the consequences as regards short-term benefits.
AFFILIATIONS

*Horten Centre, University of Zurich, Zurich,

${ }^{\S}$ Barmelweid Clinic, Barmelweid, Switzerland.

${ }^{\#}$ Clarity Research Group, Dept of Clinical Epidemiology and Biostatistics, McMaster University, Hamilton, ON, Canada.

"Dept of Medicine, University at Buffalo, Buffalo, NY, USA, and ${ }^{+}$Dept of Epidemiology, Regina Elena Italian National Cancer Institute, Rome, Italy.

\section{CORRESPONDENCE}

M.A. Puhan, Horten Centre, University Hospital of Zurich, Postfach Nord, CH-8091 Zurich, Switzerland. Fax: 41442559720 E-mail:milo.puhan@usz.ch

Received:

June 282007

Accepted after revision:

October 232007

SUPPORT STATEMENT

The present study was supported by Klinik Barmelweid (Barmelweid, Switzerland). M.A. Puhan is supported by a career award (No. 3233B0/115216/1) from the Swiss National Science Foundation (Berne, Switzerland) and $\mathrm{H}$. Schünemann is supported by a European Commission Human Factor, Mobility and Marie Curie Actions Scientist Reintegration Grant (IGR 42192; Brussels, Belgium).

\section{STATEMENT OF INTEREST}

A statement of interest for this study can be found at www.erj.ersjournals.com/misc/ statements.shtml

Online ISSN 1399-3003 


\section{METHODS}

\section{Design overview}

The methods and main results of this randomised controlled noninferiority trial (International Standard Randomised Controlled Trial Number: ISRCTN11611768) have recently been described [13]. In brief, the effects of interval exercise and high-intensity continuous exercise were compared in 98 patients with stage III-IV COPD according to the criteria of the Global Initiative for Chronic Obstructive Lung Disease. Patients with cardiovascular, musculoskeletal or neurological comorbid conditions were excluded only if they were likely to inhibit physical exercise or the performance of exercise tests. Patients were randomly assigned to either high-intensity continuous exercise or interval exercise using a computerised minimisation procedure, thereby ensuring balanced and concealed randomisation. The trial took place in a public rehabilitation clinic in Switzerland (Barmelweid Clinic, Barmelweid). The responsible ethics committee (Ethics Committee, Dept of Health and Social Security, Aarau, Switzerland) approved the study protocol, and all study participants provided written informed consent.

\section{Interventions}

Patients followed an inpatient respiratory rehabilitation programme of $\sim 3$ weeks' duration that included 12-15 exercise sessions lasting $25 \mathrm{~min}$, which was followed by individually prescribed home-based exercise (the median number of total sessions during the inpatient programme was 13 , rising to 22 when the following 2 weeks of home-based exercise was included [13]). Patients randomised to continuous exercise trained on electromagnetically braked cycle ergometers with a target workload of $70 \%$ of maximum exercise capacity. The target workload for interval exercise was 50\% (high-intensity intervals) and $10 \%$ (low-intensity intervals) of the patients' short-term maximum exercise capacity as determined by a steep ramp test. This test has been described in detail previously [13]. The work:recovery ratio was 1:2, with highintensity intervals of $20 \mathrm{~s}$ and low-intensity intervals of $40 \mathrm{~s}$. Apart from the exercise, the rehabilitation programme was identical for both groups, and included patient education, breathing therapies and optimisation of medical therapy.

\section{Outcomes}

The number of unintended breaks lasting $\geqslant 1 \mathrm{~min}$ due to dyspnoea, leg pain or other complaints was the primary outcome as regards the ability to follow assigned exercise protocols during supervised exercise sessions. This was considered to be an adequate measure of the ability to follow exercise protocols since a break represents a well defined and measurable consequence of excessive strenuous exercise. The breaks were recorded in the clinic's routine exercise diary by physiotherapists. Patients were not aware that breaks were recorded. Symptom ratings and cardiac frequency were not compared between groups as a measure of the ability to follow exercise protocols. If these parameters are used to guide adjustment of exercise intensity, as in the present study, they become ineligible as outcome measures since interventions and outcomes must be independent of each other.

As measures of the effects of respiratory rehabilitation, the selfadministered standardised German Chronic Respiratory
Disease Questionnaire (CRQ) [15, 16] and 6-min walking distance, as well as the short-time maximum exercise capacity (steep ramp test) and maximum exercise capacity (incremental exercise test), were used. The measurement of these outcomes has been described in detail previously [13].

\section{Statistical analysis}

Consequences of differing abilities to follow exercise protocols on respiratory rehabilitation outcomes

In order to enhance comparability across patients, the number of breaks per exercise session were calculated since the number of exercise sessions varied slightly across patients (median number of sessions 13 (interquartile range 12-14); 91 (93\%) patients were followed for $\geqslant 11$ sessions [13]). The patients were categorised into two groups with, on average, more than one and one or fewer breaks per exercise session. The changes in exercise capacity and HRQoL were compared between these two groups using linear regression analyses with and without adjustment for disease severity (dyspnoea, hyperinflation, hypoxaemia and baseline exercise capacity) and other characteristics (age, sex and group allocation) that might influence the association between the ability to follow exercise protocols and short-term outcomes of rehabilitation. No adjustment was made for dyspnoea for HRQoL since the dyspnoea measure is part of the CRQ (dependent variable).

\section{Subgroup analysis}

Before conducting the present analyses, the predictor variables were determined based on previous studies and plausible associations of the ability to follow exercise protocols and the outcomes of rehabilitation [7-9, 17]. However, the present trial was not designed or powered for subgroup analyses in its planning stage [17-19]. These $a$ posteriori subgroup analyses are, therefore, exploratory in nature. Subgroup analyses were restricted to the following six predictors: dyspnoea as measured by the CRQ dyspnoea domain; hypoxaemia (resting arterial oxygen tension); static hyperinflation as reflected by the resting intrathoracic gas volume (expressed as a percentage of the predicted value); 6-min walking distance; recent exacerbations ( $\leqslant 8$ or $>8$ weeks previously); and depressive symptoms ( $\leqslant 8$ or $>8$ points on the hospital anxiety and depression scale). Four of these variables (hypoxaemia, 6-min walking distance, recent exacerbation and depressive symptoms) had been used as stratification variables in the described randomisation process in order to achieve balanced groups. This indicates the $a$ priori predictions regarding their relationship to the ability to follow exercise protocols [17]. Standard parameters for COPD severity, such as forced expiratory volume in one second (FEV1) and combined indices, were not included as predictor variables as there is no evidence from earlier studies that they influence the association between the ability to follow exercise protocols and outcomes of rehabilitation.

For each of the predictor variables, patients within the interval and continuous exercise groups were divided into tertiles reflecting moderate, severe and very severe impairment. Thus patients with the lowest scores, between the 1st and 33rd percentile ( $1^{\text {st }}$ tertile), were defined as having very severe impairment, patients with scores between the 34th and 66th percentile as having severe impairment and patients with scores between the 67th and 100th percentile as having 
moderate impairment. Predictor variable data for each group are presented as mean $\pm \mathrm{SD}$. As recommended for formal subgroup analyses, the presence of subgroup effects was evaluated by testing for interaction [19, 20] and looking graphically at the trend across subgroups. A p-value of $<0.05$ was used as a very conservative cut-off for significant and clinically relevant interaction. Linear regression analyses were conducted with the primary outcome, the number of unintended breaks, as the dependent variable and group, and the predictor variable and interaction term as independent variables. The number of breaks per session was also transformed by taking the square root. With this transformation, residuals of the linear regression analyses were distributed normally. The results from the linear regression analyses were back-transformed for data presentation and facilitation of interpretation.

\section{RESULTS}

All of the 98 patients ( $66.3 \%$ male) enrolled in the trial were included in the present analysis. Their mean \pm SD age was
$68.9 \pm 9.1$ yrs, FEV1 $34.3 \pm 8.5 \%$ pred, CRQ dyspnoea at baseline score $2.94 \pm 0.97$, baseline 6-min walking distance $323 \pm 109 \mathrm{~m}$ and body mass index $24.7 \pm 6.4 \mathrm{~kg} \cdot \mathrm{m}^{-2}$, and $58.2 \%$ had cardiovascular comorbid conditions.

\section{Consequences of differing abilities to follow exercise protocols on respiratory rehabilitation outcomes}

Figure 1 shows that patients with more than one unexpected break per exercise session showed consistently lower effects of rehabilitation than those with one or fewer unexpected breaks per exercise session. For example, patients with more than one break per exercise session showed a mean improvement of only $22 \pm 69 \mathrm{~m}$ in 6-min walking distance, whereas patients with one or fewer breaks per exercise session showed a mean improvement of $53 \pm 52 \mathrm{~m}$.

Figure 2 shows the unadjusted and adjusted differences in outcomes between patients with more than one and one or fewer breaks per exercise session. In multivariable regression models, one or fewer breaks per exercise session was
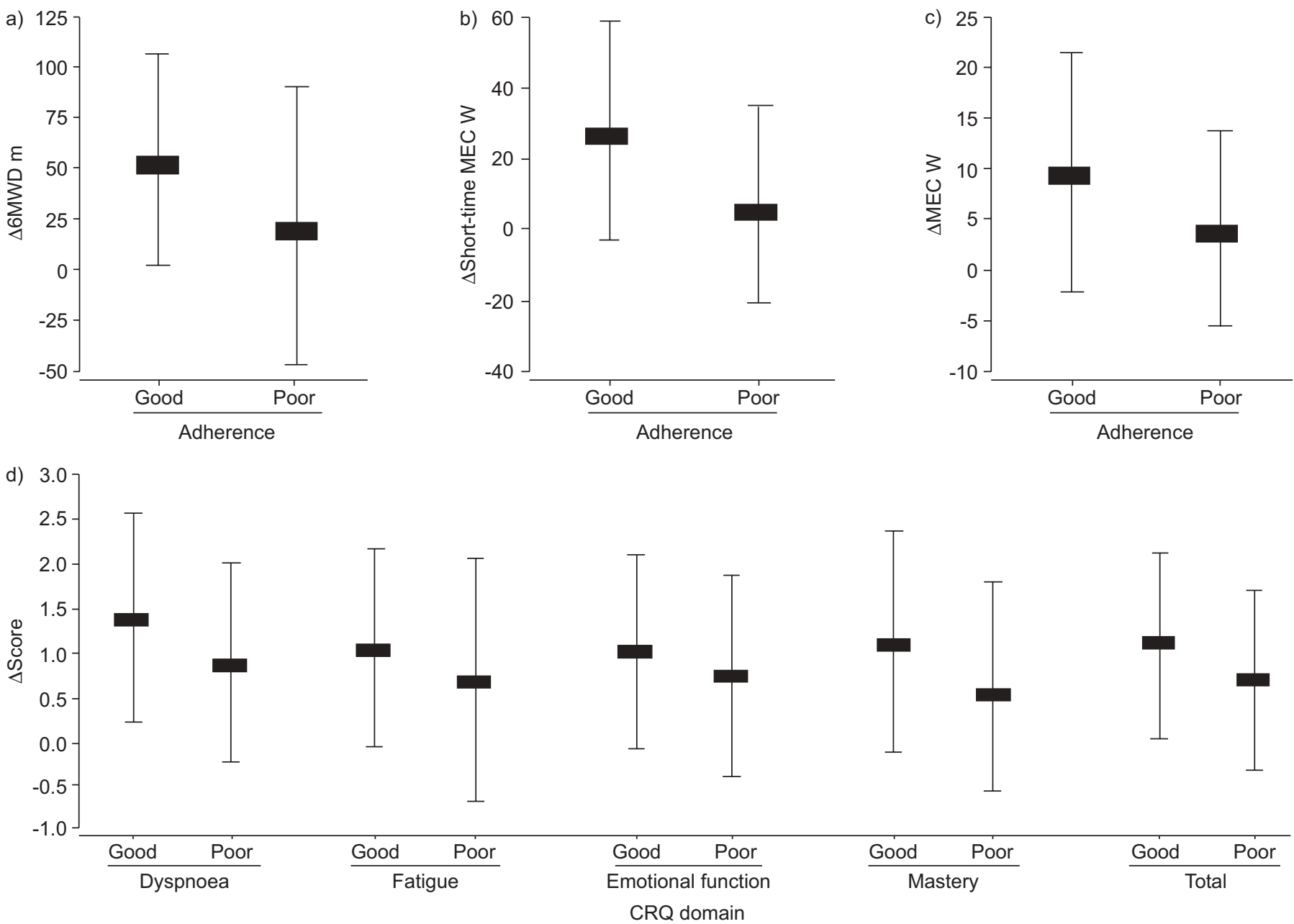

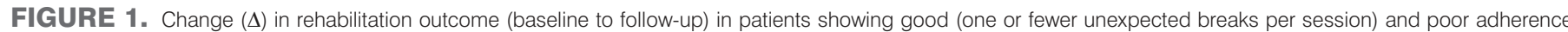

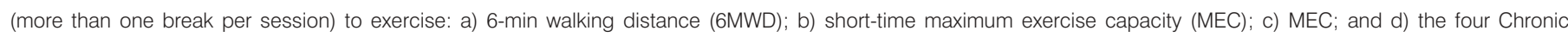
Respiratory Disease Questionnaire $(\mathrm{CRQ})$ domains. Data are presented as mean $\pm \mathrm{SD}$. 

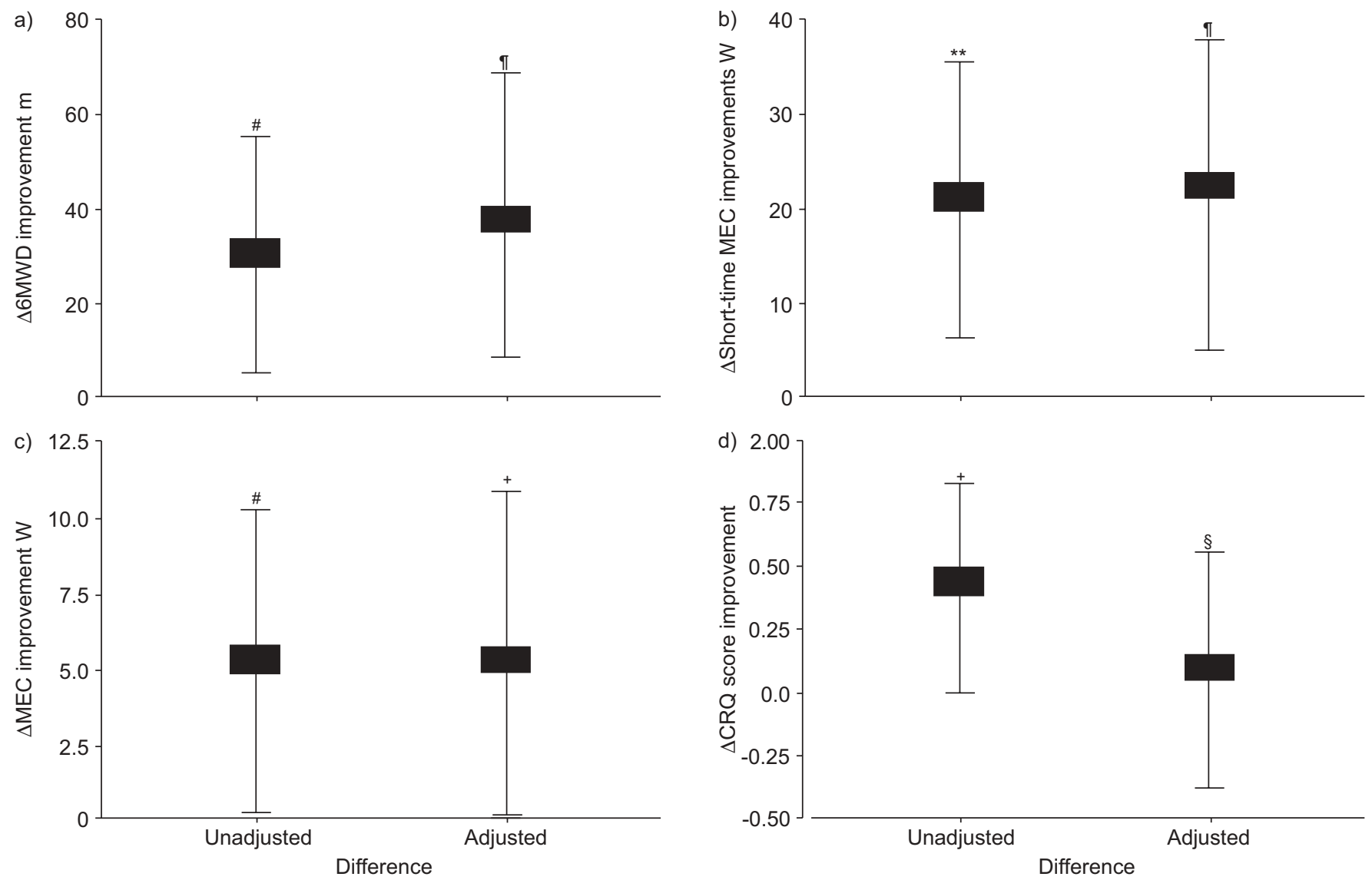

FIGURE 2. Unadjusted and adjusted mean differences $(\Delta)$ in improvement in outcome between patients with good (one or fewer unexpected breaks per session) and poor adherence (more than one break per session) to exercise: a) 6-min walking distance (6MWD); b) short-time maximum exercise capacity (MEC); c) MEC; and d) Chronic Respiratory Disease Questionnaire (CRQ) total score. Data were adjusted for age, sex, group, 6MWD, dyspnoea, hypoxaemia and hyperinflation (dyspnoea adjustment was omitted for the $\mathrm{CRQ}$ since this is one of the domains of the $\mathrm{CRQ}$ ). Vertical bars represent $95 \%$ confidence intervals. ${ }^{* *}: p<0.01 ;{ }^{\#}: p<0.02 ;{ }^{*}: p=0.01 ;{ }^{+}: p=0.05 ;{ }^{\varsigma}: p<0.62$.

independently associated with a substantially greater improvement in 6-min walking distance (38 m (95\% confidence interval (CI) 8-68; $p=0.01)$ ). Larger improvements in short-time $(p=0.01)$ and normal maximum exercise capacity $(p=0.05)$ were also observed in patients with one or fewer breaks per exercise session. Unadjusted analyses also suggested a greater effect on HRQoL ( $\mathrm{p}=0.05)$, but adjustment for disease severity reduced the difference between the two groups $(p=0.62)$.

\section{Subgroup analysis}

Table 1 shows the characteristics of the subgroups. In general, patients in corresponding subgroups of the interval and continuous exercise groups showed similar degrees of impairment. For example, patients with the least degree of hyperinflation (moderate) in the interval and continuous exercise groups showed mean intrathoracic gas volumes of $126 \pm 20 \%$ pred and $130 \pm 17 \%$ pred at study entry, respectively, whereas

\section{TABLE 1 Characteristics of the subgroups of the interval and continuous exercise groups}

\begin{tabular}{|c|c|c|c|c|c|c|}
\hline & \multicolumn{3}{|c|}{ Interval } & \multicolumn{3}{|c|}{ Continuous } \\
\hline & Moderate & Severe & Very severe & Moderate & Severe & Very severe \\
\hline Dyspnoea CRQ score & $3.78 \pm 0.50(17)$ & $2.79 \pm 0.18(15)$ & $1.86 \pm 0.47(16)$ & $4.18 \pm 0.44(17)$ & $3.03 \pm 0.36(16)$ & $1.89 \pm 0.48(17)$ \\
\hline Hyperinflation \% pred & $126 \pm 20(13)$ & $162 \pm 8(13)$ & $207 \pm 26(15)$ & $130 \pm 17(15)$ & $158 \pm 8(16)$ & $206 \pm 22(15)$ \\
\hline Arterial oxygen tension $\mathrm{mmHg}$ & $67.0 \pm 3.5(15)$ & $58.1 \pm 2.2(15)$ & $48.1 \pm 5.5(18)$ & $70.4 \pm 3.6(16)$ & $60.4 \pm 2.6(15)$ & $49.5 \pm 5.2(19)$ \\
\hline 6-min walking distance $\mathrm{m}$ & $434 \pm 56(16)$ & $309 \pm 24(16)$ & $196 \pm 50(16)$ & $458 \pm 57(17)$ & $321 \pm 27(17)$ & $211 \pm 44(16)$ \\
\hline Depression HADS score & $3.89 \pm 1.45(18)$ & $7.33 \pm 0.62(15)$ & $12.67 \pm 2.53(15)$ & $3.41 \pm 1.37(17)$ & $7.63 \pm 1.12(19)$ & $12.46 \pm 2.50(14)$ \\
\hline
\end{tabular}

Data are presented as mean \pm SD (n). For seven patients in the interval exercise group and four in the continuous exercise group, no data about hyperinflation (as reflected by intrathoracic gas volume) were available. CRQ: Chronic Respiratory Disease Questionnaire; \% pred: \% predicted; HADS: hospital anxiety and depression scale. 
the $33 \%$ of patients with the most severe hyperinflation showed mean intrathoracic gas volumes of $207 \pm 26 \%$ pred and $206 \pm 22 \%$ pred, respectively. Patients in the interval exercise group with moderate and severe dyspnoea exhibited somewhat lower mean dyspnoea scores than those in the corresponding subgroups of the continuous exercise group. Patients with the most severe dyspnoea, however, showed similar dyspnoea scores. In the interval exercise group, 29 $(60.4 \%)$ patients had experienced a recent exacerbation compared with $30(60.0 \%)$ in the continuous exercise group.

Figure 3 shows the subgroup analyses. Patients with moderate dyspnoea tolerated interval and continuous exercise similarly (difference in number of unexpected breaks per exercise session of $0.1(95 \%$ CI $-0.3-0.5))$. Patients with more severe dyspnoea at study entry in the continuous exercise group required more breaks than those in the interval exercise group (difference $0.4(95 \%$ CI $-0.2-1.1)$ for patients with severe dyspnoea and 0.8 (95\% CI 0.0-1.6) for patients with very severe dyspnoea). Testing for the subgroup effect (interaction for group $\times$ dyspnoea) showed a $\mathrm{p}$-value of 0.08 .

Similar results were observed for the subgroup analyses of hypoxaemia, hyperinflation and 6-min walking distance. Again, the more severe the impairment the greater the difference in the number of breaks. This trend was more pronounced and significant for hypoxaemia $(p=0.04$ for subgroup effect) than for hyperinflation $(p=0.15$ for subgroup effect) and 6-min walking distance ( $p=0.17$ for subgroup effect). The two remaining subgroup variables, recent exacerbation and depressive symptoms, were not related to the number of breaks $(p=0.49$ and $p=0.59$, respectively, for subgroup effect).

\section{DISCUSSION}

The present study shows that the ability to follow exercise protocols plays an important role as a predictor of enhanced short-term benefits from respiratory rehabilitation. Patients with one or fewer major breaks per exercise session showed significantly greater improvements in exercise capacity than those with more than one break. Improvements in HRQoL were significantly greater in the unadjusted analyses alone. The present study also suggests that the degree of impairment, as represented by the degree of dyspnoea, exercise capacity, hyperinflation and hypoxaemia, may influence how well patients can follow interval compared to continuous exercise programmes. However, this subgroup analysis was not preplanned, and only some of the differences were significant.

One strength of the present analysis is that the data come from a randomised trial with high quality methods. Another is the implementation of the trial in an everyday clinical setting in typical COPD patients. In addition, predictor variables were determined that were biologically or clinically plausible before performing any subgroup analyses and that had, in part, already been identified as possible effect modifiers during the planning phase of the trial. In addition, recommended standards were followed for the exploration of subgroup effects using interaction terms in regression analyses [19].

A major limitation was that the present trial was, a crux for most subgroup analyses, insufficiently powered for the subgroup analyses. The sample size calculation was based

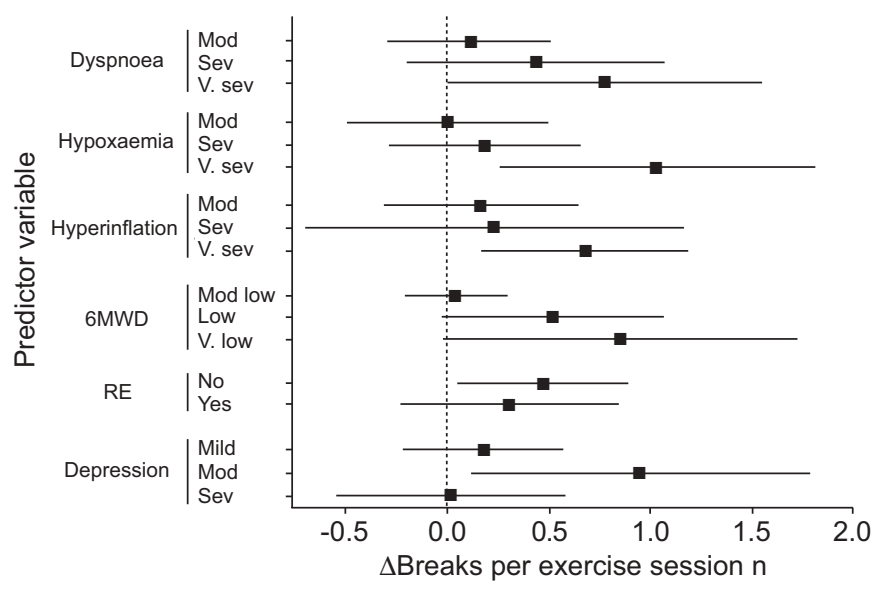

FIGURE 3. The six subgroup analyses. Data are presented as difference $(\Delta)$ in number of breaks per exercise session between the interval and continuous exercise groups. Positive differences indicate more breaks with continuous exercise. Horizontal bars represent 95\% confidence intervals. The p-values for subgroup effects stem from the interaction term (predictor variable $\times$ subgroup) in the multivariable regression analyses: dyspnoea (Chronic Respiratory Disease Questionnaire; $C R Q$ score): $p=0.08$; hypoxaemia: $p=0.04$; hyperinflation: $p=0.15$; 6-min walking distance (6MWD): $p=0.17$; recent exacerbation $(R E): p=0.49$ depressive symptoms (depression): $p=0.59$. mod: moderate; sev: severe; v.: very.

only on the main comparison of the trial [13]. In addition, one limitation of restricting subgroup analyses to six variables is that more plausible predictor variables may exist. However, restricting the number of predictor variables (six) decreased the risk of finding subgroup effects due to chance [18]. Finally, medical emergencies and accidents were recorded only during exercise sessions (none were observed), but not the reasons for each major break.

To the present authors' knowledge, the current trial is the only COPD trial that has assessed the consequences of the ability to follow specific exercise protocols on short-term outcomes. The focus on this aspect is important for several reasons. As the present analysis has shown, patients show more short-term benefit from rehabilitation if they can follow the exercise protocol without major breaks. Differences in improvements in exercise capacity were substantial and not affected much by adjustment. In contrast, HRQoL differed between groups in the unadjusted analyses alone. Adjustment for baseline hyperinflation and other parameters moderated between-group differences, which indicates their effect on improvements in HRQoL.

Experiencing a tolerable exercise protocol during the supervised initiation phase of exercise may also be important in the long term. Many COPD patients exercise for the first time after a period of physical inactivity. In order to facilitate this initiation, it is important that patients tolerate the exercise well and perceive short-term benefit, since this will probably improve adherence to training programmes in the long term. This aspect has not been addressed, but future studies may provide information in this regard. In addition, the present parameter for the ability to follow exercise protocols, the mean number of major breaks, was rather simplistic. This simple parameter makes it attractive for trials and practice, but does not offer a more differentiated analysis of exercise tolerance. 
Future trials on exercise in COPD should evaluate whether or not better measures of the ability to follow exercise can be developed. Thereby, more would be learnt about this important aspect for clinical practice.

Previous studies have suggested that interval exercise is particularly well tolerated by patients with severe COPD [79]. The low-intensity intervals limit dynamic hyperinflation, which is most pronounced in severely impaired COPD patients. Based on these studies, parameters for impairment were selected that are readily available in clinical practice. As noted above, the present study was too small to show significant subgroup effects, and it is possible that the results are due to chance. However, there is no prior evidence addressing this point from respiratory rehabilitation trials. A trend was found that the most severely impaired patients who enter rehabilitation tolerate interval exercise better than continuous exercise, but additional larger studies are needed to confirm these findings. Investigators commonly use more lenient thresholds, such as $\mathrm{p}<0.1$ and $\mathrm{p}<0.2$, for interaction effects. Subgroup effects were considered significant if $\mathrm{p}$ values for interaction terms were $\leqslant 0.05$ in the present study. The decision to use this conservative threshold was based on the number of predictor variables. If a higher threshold had been used for significance, dyspnoea would also have been a significant predictor.

It is important that subgroup analyses are planned in advance for future trials, and that such trials are powered accordingly. Thereby, more would be learnt about the variables available in clinical practice that guide the selection of exercise protocols. For clinicians, it would be attractive to use these parameters in combination. For example, a simple score could be developed containing parameters, such as dyspnoea, hyperinflation, hypoxaemia and exercise capacity, for the prediction of tolerance of specific exercise protocols.

\section{Conclusion}

The present study has shown that it is important to select an exercise protocol that chronic obstructive pulmonary disease patients are able to follow. If patients need at least one major break lasting $\geqslant 1 \mathrm{~min}$ per exercise session, they are less likely to benefit from respiratory rehabilitation, at least in the short term. Interval and continuous exercise are prudent choices for Global Initiative for Chronic Obstructive Lung Disease stage III-IV chronic obstructive pulmonary disease patients if they show moderate impairment due to increased dyspnoea, hypoxaemia, hyperinflation or moderately reduced exercise capacity. If patients are severely impaired, interval exercise is likely to be better tolerated than continuous exercise.

\section{ACKNOWLEDGEMENTS}

The authors would like to thank all of the physiotherapists and physicians for their assistance in the conduct of the present study. They also thank A. Kessels for advice on the statistical analyses.

\section{REFERENCES}

1 Skeletal muscle dysfunction in chronic obstructive pulmonary disease. A statement of the American Thoracic
Society and European Respiratory Society. Am J Respir Crit Care Med 1999; 159: S1-S40.

2 Component 3: Manage stable COPD. In: Global Initiative for Chronic Obstructive Pulmonary Disease. Global Strategy for the Diagnosis, Management, and Prevention of Chronic Obstructive Pulmonary Disease, 2006. Edgewater, MCR Vision, Inc., 2006; pp. 47-61. http:/ /www.goldcopd.com/ download.asp?intId=379 Date last updated: November 2006. Date last accessed: 20 February 2007.

3 British Thoracic Society. Managing stable COPD. Thorax 2004; 59: Suppl. 1, 39-130.

4 Lacasse Y, Brosseau L, Milne S, et al. Pulmonary rehabilitation for chronic obstructive pulmonary disease. Cochrane Database Syst Rev 2004; 4: CD003793.

5 Puhan MA, Scharplatz M, Troosters T, Steurer J. Respiratory rehabilitation after acute exacerbation of COPD may reduce risk for readmission and mortality - a systematic review. Respir Res 2005; 6: 54.

6 Puhan MA, Schunemann HJ, Frey M, Scharplatz M, Bachmann LM. How should COPD patients exercise during respiratory rehabilitation? Comparison of exercise modalities and intensities to treat skeletal muscle dysfunction. Thorax 2005; 60: 367-375.

7 Sabapathy S, Kingsley RA, Schneider DA, Adams L, Morris NR. Continuous and intermittent exercise responses in individuals with chronic obstructive pulmonary disease. Thorax 2004; 59: 1026-1031.

8 Vogiatzis I, Georgiadou O, Golemati S, et al. Patterns of dynamic hyperinflation during exercise and recovery in patients with severe chronic obstructive pulmonary disease. Thorax 2005; 60: 723-729.

9 Vogiatzis I, Nanas S, Kastanakis E, Georgiadou O, Papazahou O, Roussos C. Dynamic hyperinflation and tolerance to interval exercise in patients with advanced COPD. Eur Respir J 2004; 24: 385-390.

10 Arnardottir RH, Sorensen S, Ringqvist I, Larsson K. Two different training programmes for patients with COPD: a randomised study with 1-year follow-up. Respir Med 2006; 100: 130-139.

11 Coppoolse R, Schols AM, Baarends EM, et al. Interval versus continuous training in patients with severe COPD: a randomized clinical trial. Eur Respir J 1999; 14: 258-263.

12 Kaelin MB, Barnard K, Swank A, Adams K, Ponto A. Results of 6 minute ambulation and MET tolerance of patients with severe chronic obstructive pulmonary disease (COPD) utilizing 2 different aerobic training regimes: interval training versus continuous training. JEP 1999; 2: A24.

13 Puhan MA, Busching G, Schunemann HJ, VanOort E, Zaugg C, Frey M. Interval versus continuous high-intensity exercise in chronic obstructive pulmonary disease: a randomized trial. Ann Intern Med 2006; 145: 816-825.

14 Vogiatzis I, Nanas S, Roussos C. Interval training as an alternative modality to continuous exercise in patients with COPD. Eur Respir J 2002; 20: 12-19.

15 Puhan MA, Behnke M, Frey M, et al. Self-administration and interviewer-administration of the German Chronic Respiratory Questionnaire: instrument development and assessment of validity and reliability in two randomised studies. Health Qual Life Outcomes 2004; 2: 1. 
16 Puhan MA, Behnke M, Laschke M, et al. Selfadministration and standardisation of the Chronic Respiratory Questionnaire: a randomised trial in three German-speaking countries. Respir Med 2004; 98: 342-350.

17 Puhan MA, Busching G, VanOort E, Zaugg C, Schunemann $\mathrm{HJ}$, Frey M. Interval exercise versus continuous exercise in patients with moderate to severe chronic obstructive pulmonary disease - study protocol for a randomised controlled trial [ISRCTN11611768]. BMC Pulm Med 2004; 4: 5.
18 Oxman AD, Guyatt GH. A consumer's guide to subgroup analyses. Ann Intern Med 1992; 116: 78-84.

19 Rothwell PM. Treating individuals 2. Subgroup analysis in randomised controlled trials: importance, indications, and interpretation. Lancet 2005; 365: 176-186.

20 Pocock SJ, Assmann SE, Enos LE, Kasten LE. Subgroup analysis, covariate adjustment and baseline comparisons in clinical trial reporting: current practice and problems. Stat Med 2002; 21: 2917-2930. 